\title{
LA VICTIMIZACIÓN DE MADAME MERLE IN THE PORTRAIT OF A LADY Y LA SOCIEDAD EUROPEA COMO AGENTE OPRESOR
}

MADAME MERLE'S VICTIMIZATION IN THE PORTRAIT OF A LADY AND THE EUROPEAN SOCIETY AS OPPRESSIVE AGENT

Marina Filgueira Figueira Universidad de A Coruña

\section{Resumen:}

Este estudio se centra en uno de los personajes principales de la novela The Portrait of a Lady DE Henry James y el papel de la sociedad europea en el proceso de victimización femenina. Este análisis pretende ofrecer una nueva perspectiva de la novela y la representación del malvado personaje de Madame Merle. Su metodología consiste, por un lado, en un enfoque cronológico $\mathrm{y}$ por el otro, un enfoque sincrónico. La y por el otro, un enfoque sincrónico. La contradicción entre la información recogida tras los análisis nos ha permitido llegar a la conclusión de que el personaje de Madame Merle debe analizarse victimización femenina.

Palabras claves:

Madame Merle, victimización, sociedad novela.

\section{INTRODUCCIÓN}

Henry James nace en Nueva York y es hijo de uno de los más célebres intelectuales en América durante el siglo XIX. Entre los amigos de Henry James Sr. se encuentran figuras como Ralph Waldo Emerson, Henry David Thoreau y Nathaniel Hawthorne. Bajo este influjo intelectual, crece la figura de Henry James, quien pronto no sólo se dejará influir por esto, sino también por sus viajes continuos por Europa. Durante toda su juventud, visitó ciudades como Génova, Bolonia, Londres, París, y Bonn. Gracias a su formación literaria leyó no sólo la obra de los grandes autores norteamericanos, sino también los clásicos europeos, y así se empapó de la literatura inglesa, la francesa la alemana e incluso leyó literatura rusa. Tras vivir durante un tiempo en París, se instaló en Londres. James encuentra en la nueva Europa su paraíso de inspiración literaria, y durante estos años, escribe sus primeras novelas en las que retrata el modo de vivir de los americanos, que como él, han hallado en Europa su nuevo hogar. Sin embargo, nunca llegó a sentirse plenamente integrado en su país de adopción, a la vez que tampoco sentía América como verdadero hogar. Estas contradicciones interiores le llevaron a crear personajes con grandes complejidades, que compartían con él este sentimiento. Por otro lado, y como veremos a continuación, Henry James es un maestro de la caracterización femenina, y muchos de sus personajes femeninos sorprenden no sólo por esa riqueza, sino también por la sensibilidad y entendimiento con el que fueron creados. Uno de estos personajes femeninos, de nacionalidad americana y afincado en Europa -como no podía ser de otro modo- es el objeto de este estudio que, como inmediatamente se explica, ha sido en parte olvidado por la crítica.

Por otra parte, creemos que la obra de Henry James, The Portrait of a Lady (1881), resulta también atractiva desde otro punto de vista, ya que esconde muchos elementos útiles dentro del debate planteado por los estudios femeninos actuales. Por todos es conocida la preocupación de este autor por la figura femenina y tal como sugiere Elizabeth Allen en A Woman's Place in the Novels of Henry James, "[...] the attempt to reconcile the contradiction of woman's existence, both as sign and as conscious subject, is central to many of James's major novels" (cit. en Foss 1995: 253). El estudio clasicista de la obra ha planteado cuestiones como el punto de vista en la narración o la caracterización de la protagonista como elementos centrales a su estudio. Sin embargo, bajo la luz de los nuevos estudios de género, obras como la que nos ocupa pueden ofrecer un sinfín de posibilidades a profesoras/es y alumnas/os que encontrarán en este tipo de análisis un nuevo campo de trabajo. Así, este artículo pretende ofrecer una nueva posibilidad de acercamiento a obras que pertenecen al canon literario seguido por la mayoría de las universidades europeas y que pueden aportar nuevas líneas de investigación bajo el prisma de los estudios de género. El concepto de victimización 
femenina se toma pues aquí como base de estudio para el análisis de esta obra y pretende mostrar un lado poco explorado de uno de los personajes femeninos más misteriosos de la novela norteamericana con trasfondo europeo.

El personaje de Madame Merle no ha sido suficientemente estudiado y analizado por los críticos a lo largo de estos años. El interés creado por la crítica se ha centrado en los dos personajes principales de la novela, Isabel Archer y Gilbert Osmond, olvidando el tercer elemento en el triángulo amoroso de la novela. William Sayres explica que "[...] studies which single [Madame Merle] out for special consideration are few and far between" (1992: 231-232). Sin embargo, este personaje ha sido considerado en alguna ocasión como "the most perfect creation" (Beach 1918: 207), y por ello, uno de los más difíciles de analizar. El mismo Henry James reconoce en sus Notebooks que "[...] the whole matter of Madame Merle is [...] a very ticklish one -very delicate and difficult to handle" (cit. en Stafford 1986: 118). Por ello, este estudio ambiciona descubrir un lado oculto de este personaje que tal y como se pretende demostrar a lo largo de estas páginas, sufre la marginalización no sólo de la crítica actual, sino también un proceso de discriminación por parte del narrador y por supuesto, por parte de muchos de los personajes de la novela que pertenecen, o se han aclimatado, a una sociedad europea, de la que quizá reciben a su vez una presión social. El perfil malvado de Madame Merle se verá aquí enriquecido por otros roles que este personaje asume a lo largo de la novela, puesto que tal y como explica Stafford “Madame Merle's is much more complex, more human, more demanding, more interesting" (1986: 121), y aquí se intentará demostrar que este personaje se puede estudiar desde una perspectiva distinta. Para ello, en primer lugar se propone un análisis cronológico de la información que obtenemos a lo largo de la lectura de la obra; posteriormente, un estudio sincrónico de dicha información, nos permitirá arrojar una nueva luz sobre la figura de Madame Merle, en la que el papel que la sociedad europea de principios del siglo XX juega un papel importante.

El personaje de Madame Merle, como se ha dicho anteriormente, es difícil de analizar por la complejidad que entraña. En este trabajo se estudiarán y analizarán los diferentes roles que su personaje ejecuta a lo largo de la novela, así pues, en primer lugar, observamos que Madame Merle es una mujer americana que vive en Europa entre una sociedad que la ha acogido tras dejar atrás su país de origen, al igual que Henry James, aunque como veremos más adelante, es esta sociedad la culpable de muchos de sus sufrimientos. Madame Merle actúa a su vez como amante, como madre y como amiga a lo largo de la novela. Como mujer, Madame Merle es descrita física y psicológicamente en su primera aparición en el capítulo XVIII. De hecho, se la presenta como una dama. En estas páginas se nos ofrece una descripción de sus cualidades, hábitos y comportamiento. Leemos sobre su destreza con el piano, su dominio del francés, su gusto por la pintura, la literatura y el bordado. Su pasión por dar largos paseos y por contestar a su correspondencia, ocupan el día de nuestra heroína, tal y como lo hacían las mujeres de su época en Europa. Socialmente, sabemos que es una mujer distinguida y conocida por la alta sociedad, aunque se nos aclara que no pertenece a la aristocracia. También se nos menciona su edad "forty years old" (James 1984: 226) ${ }^{1} \mathrm{y}$ físicamente se nos dice que no era "pretty, though her expression charmed" (PL: 226).

El segundo papel que Madame Merle desarrolla a lo largo de la novela y se analiza aquí es el de amante. Como mujer, Madame Merle experimenta sentimientos, ama y se deja amar, en este caso por diversos hombres². Sabemos que ha sido amada por Ralph Touchett, que ha estado casada con un caballero suizo y que ha sido amante de Gilbert Osmond. El amor ocupa un lugar importante en su vida, ha hecho muchas cosas por amor, y a lo largo de toda la historia, desencadena otras muchas por esa lucha que nace del amor. Más tarde examinaremos este lado al tratar de explicar el proceso de victimización que sufre este personaje.

Por otro lado, observamos el tercer rol asumido por Madame Merle, el de madre. Casi al final de la novela se nos revela que Madame Merle tiene una hija nacida de modo ilegítimo fruto de su amor secreto con Gilbert Osmond. Así descubrimos que Madame Merle es la madre de Pansy, y que -aunque se ha visto obligada a perder sus derechos sobre su hija -ella ha seguido de cerca -y de algún modo, cuidando a Pansy. De hecho, siempre se ha preocupado por su educación, su futuro, su matrimonio, entre otras muchas cosas. Este papel maternal también será clave en nuestro intento por demostrar el sufrimiento que encierra el oculto rol de Madame Merle.

Para terminar con estos cuatro primeros roles, diremos que este personaje también desarrolla el papel de amiga. Al principio de la historia, Madame Merle es presentada a Isabel Archer como amiga de Mrs. Touchett, tía de la protagonista. Pero aunque se la presente como amiga personal de Mrs. Touchett, realmente se la considera amiga de la familia, como su presencia en momentos tan duros como ante la muerte de $\mathrm{Mr}$. Touchett lo demuestra. Más tarde, se convertirá en la compañía más fiel a Isabel, y entre estos dos personajes femeninos, centrales en la novela, parece nacer una verdadera amistad. Juntas pasan horas y comparten secretos y conversaciones vitales, en esos momentos, parece imposible augurar el engaño del que será víctima Isabel, y en cierta forma, el lector, que comparte la misma información que la protagonista, al menos en

1 James, H., The Portrait of a Lady, 1881, ed. Geoffrey Moore, London: Penguin, 1984. En adelante PL.

2 Aunque debemos señalar el punto de vista de Melissa Solomon en su artículo "The Female World of Exorcism and Displacement (or, Relations between Women in Herny James's Nineteenth-Century The Portrait of a Lady", Studies in the Novel 28.3 (1996), pp. 395-413, donde señala la relación lésbica entre Madame Merle e Isabel Archer en la novela. 
la primera parte de la novela. Si observamos el comportamiento de Madame Merle en este cuarto papel, podemos incluso asimilar algún aspecto de su rol como madre al de amiga. Madame Merle, en ocasiones, se comporta como una madre con Isabel una vieja amiga que la superprotege y la aconseja. Siempre parece estar al cuidado de la joven americana, parece preocuparse por su bienestar, parece amarla como una verdadera madre lo haría.

Hasta este punto, todo lo que hemos comentado sobre Madame Merle son aspectos aparentemente positivos, puesto que siguiendo la focalización de información que el narrador realiza en la novela, el lector desconoce, al igual que lo hace la protagonista, el verdadero plan del personaje malvado. El punto de vista que observamos a lo largo de las dos primeras partes de la novela es el que compartimos con la protagonista, Isabel Archer, tal y como comenta Martha Collins “James's primary concern, throughout the preface, was Isabel's consciousness" (1976: 142). Sin embargo, si seguimos analizando la información, descubrimos la verdadera naturaleza de Madame Merle, su lado secreto. A partir del capítulo XL Madame Merle se consolida como uno de los personajes oscuros de la historia, es ella la que planea y prepara la unión fatal entre Isabel y Gilbert Osmond, que será la que desencadene la tragedia de Isabel. Aunque el personaje masculino es por supuesto el otro 'culpable', es Madame Merle quien traiciona la amistad que Isabel y ella habían cultivado, no sólo mintiendo sobre su pasado, quizá para evitar cualquier tipo de conexión entre Gilbert, Pansy y ella, sino que también elabora un tergiversado plan cuyo único fin sólo favorece los intereses de Madame Merle y de los suyos, pero no a la felicidad de la heroína. El narrador nos la describe como una mujer encantadora, el lector no puede imaginar que aquella mujer del principio de la historia que tocaba maravillosamente el piano, que disfrutaba de largos paseos junto a Isabel, y que hablaba de aquel modo sobre Gilbert Osmond y Pansy sea finalmente la figura malvada de la trama. Tanto el lector como Isabel sólo reciben señales favorables de Madame Merle. Es cierto que por parte de algunos personajes de la historia se escuchan comentarios acerca de la poca fiabilidad de este personaje. Sin embargo, tanto el lector como Isabel confían desde el principio en ella. Es precisamente esto lo que facilita que Madame Merle pueda acometer su plan, de otro modo, le sería imposible llevarlo a cabo. La confianza de la protagonista, y en cierto modo, del lector, son elementos necesarios para que pueda mostrarse finalmente como es. La dosificación de información que el lector recibe empieza a diferenciarse de la que Isabel obtiene a medida que avanza la novela, así lo describe Collins:

Gradually, [the narrator] mingles his historical perspective with the observations and reflections of Isabel and the satellites $[\ldots]$ The method changes in the second section (chaps. 18-35): when Madame Merle and Osmond are introduced, James begins withholding significant information and allows us to follow Isabel's perceptions more closely. At the same time, through the narrator's hints and revelations and frequent use of the satellite points of view, James keeps us considerably more aware than Isabel [...] In the final third [section], the great action is over, Isabel is settled and James has only to reveal -an Isabel to discoverthe extent of prior deceptions and the effect of prior deceptions. (Collins 1976: 143)

El lector atisba la crueldad del personaje en cuanto conoce el plan secreto de Madame Merle en relación con la unión entre Isabel y Osmond, y somos a su vez, conocedores de la relación que aquella mantuvo con este último años atrás. Sin embargo, Isabel recibe esta preciada información demasiado tarde cuando en el capítulo LI, Countess Gemini se lo hace saber. Hemos de hacer notar que sin embargo, ésta ya presuponía alguna mala intención en Madame Merle en el capítulo XLIX.

Como hemos visto hasta aquí, el personaje de Madame Merle ofrece una gran riqueza y es interesante e importante el proceso que se siga para acercarse a este personaje. Aquí se proponen dos diferentes formas de aproximación: en primer lugar, ofrecemos la perspectiva cronológica en la que juzgaremos al personaje siguiendo la información que como lectores obtenemos por parte del narrador y de la propia historia paso a paso, mientras que en segundo lugar, optamos por un análisis sincrónico en el que valoramos al personaje tras haber conocido todos los detalles que la historia arroja. Es al menos interesante el resultado que obtenemos al comparar ambos estudios, puesto que debemos admitir que nuestra percepción de Madame Merle puede variar según la forma en la que se la estudie.

\section{EL LADO PERVERSO Y CONOCIDO DE MADAME MERLE: ESTUDIO CRONO- LÓGICO DE LA INFORMACIÓN}

La perversidad de Madame Merle nace de nuestra apreciación como lectores a partir del capítulo XXII, pero Isabel no es hasta el capítulo LI cuando descubre la realidad de su antigua amiga. Collins ilustra magistralmente esta dosificación de la información: "[r]aising curtains on scenes that Isabel does not witness and looking into the corners of other characters' minds, James Keeps us more aware than Isabel of the source of the mystery. But because the revelation is gradual and incomplete, we share Isabel's victimization at the same time that we are partially aware of it" (Collins 1976: 150).

\footnotetext{
3 En relación a la dosificación de información que caracteriza la técnica narrativa de Henry James en The Portrait of a Lady y posteriormente muchas de sus otras novelas, podemos señalar un dato importante que ya apuntaba de modo suscinto Adrian Poole en su artículo "Dying before the End: The Reader in The Portrait of a Lady", Yearbook of English Studies, 26 (1996), pp. 143-53. La obra que nos ocupa fue publicada como coleccionables durante catorce meses en el Reino Unido en Macmillan's Magazine y en Estados Unidos en Atlantic Monthly. El mismo Poole nos dice que "[i] $t$ is easy to suppose that the kind of fiction James wanted to write was entirely at odds with the exigencies and possibilities of serial form" (1996: 144).
} 
Si comenzamos a analizar la figura de Madame Merle de modo cronológico, es decir, siguiendo la información que recibimos por parte del narrador, de los diálogos de los personajes, de sus opiniones y a partir de los hechos que tienen lugar a lo largo de la novela, el comportamiento de Madame Merle debe ser considerado como el del personaje malvado de la novela, o al menos, el de uno de ellos. La clave de esta identificación del mal con Madame Merle radica en que el lector 'sufre' junto a Isabel el engaño, puesto que "[...] like Isabel, we are given only the pieces of a puzzle which we must fit together ourselves( the differences being that we are given more pieces, and given them sooner" (Collins 1976: 151).

Analicemos pues las señales que recibimos acerca del carácter malévolo de nuestra protagonista. El primer indicio de su enmascarada naturaleza es su propio nombre ${ }^{4}$ 'Merle' término francés que en inglés significa 'blackbird' (mirlo en español) evoca varios significados, muchos de ellos contradictorios, o al menos, ambiguos. Soñar con un mirlo se interpreta como un mal presagio, mientras que si el mirlo está cantando y/o volando, es anuncio de buena fortuna. Sin embargo, abundan más los aspectos negativos asociados a este pájaro. El color negro de su plumaje apunta también el ocultismo, la separación, o la tristeza. Puede determinar todo lo que está escondido y velado: muerte, noche. Aunque también se le asocia con otros elementos positivos como la seriedad, la nobleza, la sofisticación, la elegancia, o el poder. Sin embargo, podemos ir más lejos, puesto que según el estudio de la simbología heráldica:

The blackbird in ancient times was called Medula, because it sang rhythmically. Others say that it was called Merula, because it flew on its own. It represents those tainted by the blackness of sin; a symbol of temptation [. . ] It is a mark of Cadhose tainted by the ble and was used as the symo on his inability to inherit, and walk on, his ancestral lands. May signify one who had to subsist by virtue and merit, not inheritance; also denotes promptness. The legend of the martlet is most appealing but readers should determine their own interpretation, as history seems to be confused about the dubious origins and myths of this marvellous bird. (Heraldryclipart.com)

Por otro lado, para muchos lectores existe una similitud entre Madame Merle y Marquise of Merteuil, el personaje malvado de la obra Les Liaisons Dangereuses (1782) de Pierre Choderlos de Laclos, que hace presagiar las malvadas intenciones de la mujer con relación a Isabel.

Existen otras señales que avisan del lado oculto de Madame Merle si seguimos los comentarios de los personajes de la novela, así el que un personaje como Ralph

4 Como ya se ha comentado anteriormente, esta obra fue inicialmente publicada por entregas durante más de un año, desde octubre de 1880 hasta noviembre de 1881 en el Reino Unido, y debemo señalar en relación al nombre de Madame Merle, que en la entrega de febrero de 1881, su nombre es 'Geraldine', mientras que en septiembre y finalmente en la publicación como libro, su nombre cambia a 'Serena'.
Touchett desconfíe de ella es más que significativo. Ya en el capítulo XVIII muestra su desagrado ante su figura al describirla como demasiado perfecta para ser auténtica ante los incesantes halagos por parte de Isabel "[s]he does everything beautifully. She is complete" (PL: 230), y en el capítulo XXIII insiste diciendo: "[...] she pushes the search for perfection too far [...] She's too good, too kind, too clever, too learned, too accomplished, too everything. She is too complete in a word" (PL: 301). En contraste con lo que opina Ralph, Mrs. Touchett en el capítulo XIX afirma que "[s]he is incapable of a mistake; she is the most tactful woman I know [...] Serena Merle hasn't a fault [...] She's one of the most brilliant women in Europe [...] You won't discover a fault in her (PL: 246), aunque esta insistencia en la perfección de Madame Merle nos hace sospechar de algún modo.

Los capítulos que siguen nos muestran como surge y se afianza la amistad entre Serena Merle e Isabel, somos testigos de las confidencias de la joven, y atisbamos un misterio alrededor de la figura de Madame Merle, quien dosifica la información acerca de su pasado de un modo un tanto sospechoso. Isabel obtiene tan sólo la información que Madame Merle desea darle y por ella sabemos que es una mujer solitaria que esconde un pasado. Sin embargo, más adelante el narrador y su focalización varían y aportan más datos sobre ella. Cuando Madame Merle en el capítulo XXII se encuentra con Gilbert Osmond en el convento ${ }^{5}$ en el que la hija de éste está internada, descubrimos la maldad y traición de este personaje, y evidentemente empezamos a sospechar sobre la relación que le une a este hombre tal y como nos dice el narrador: "[i]n the manner and tones of these two persons, on first meeting at any juncture, and especially when they met in the presence of others, was something indirect and circumspect, as if they had approached each other obliquely and addressed each other by implication" (PL: 291). Aunque la conversación que ellos mantienen también arroja muchas pistas sobre una posible relación amorosa en el pasado "[h]ow long is it since you made a new acquaintance?' ‘I don't think I've made any since I made yours."’ (PL: 290)6 También es relevante el interés que Madame Merle muestra por Pansy, la hija de Gilbert, y el poder que parece ejercer sobre ésta tal y como las propias palabras de Osmond hablando con las hermanas del convento nos hacen ver "[ $\mathrm{t}]$ his lady's a great friend of ours [...] We've much faith in her judgement, and she'll help me to decide whether my daughter shall return to you at the end of the holidays (PL: 285). Otro elemento que nos avisa de sus perversos planes son sus palabras al decirle a Pansy que no eche de menos a ninguna de las hermanas del convento, puesto que "[...] 'perhaps some day' added Madame Merle 'you'll have another mother"' (PL: 287). Y es precisamente por

5 No debemos dejar escapar el sitio donde se encuentran, y las connotaciones que dicho lugar tiene. Recordemos, a proposito, la escena en que Hamlet manda a Ofelia a la "nunery" (III,i), palabra que en la jerga de la Inglaterra protestante de Shakespeare significaba tanto convento como prostíbulo. 
medio de su conversación con Osmond en el capítulo XXII que conocemos su plan de unir a Isabel a Gilbert para su propio provecho:

'You're always doing something for your friends' [...] 'I don't however, make a crime of that,' she added [...] 'I never knew a person whose life touched so many other lives.' 'What do you call one's life?' asked Madame Merle [...] 'I call your life your ambitions,' said Osmond [...] 'My ambitions are principally for you,' aid

Florence.' (PL: 288,289)

A partir de este momento, los lectores somos poseedores de una información que Isabel aún no disfruta y que será la clave de la trama, el lector se enfrenta a una nueva fase en su lectura, a partir de este momento, su cometido será ver quién es engañado y quién no, pero sobre todo, cómo Serena Merle ejecuta su plan. Son varios los personajes que descubren el plan de Madame Merle, la condesa Gemini, hermana de Gilbert Osmond, no duda en hacerle ver que conoce su plan en el capítulo XXV:

'My dear,' [Countess Gemini] then observed to [Madame Merle], 'you'll excuse me if I don't congratulate you!' 'Very willingly, for I don't in the least know why you should, 'Haven't you a little plan that you think rather well of?' And the Countes

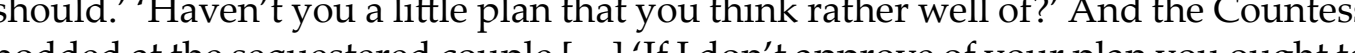
nodded at the sequestered couple $[. .$.$] 'If$ know it in order to appreciate the danger of my interfering with it. Madame Merle looked as if she were ready to admit that there might be something in this; but in a moment she said quietly: 'You think me more calculating than I am.' [...] 'You're capable of anything, you and Osmond. I don't mean Osmond by himself, and I don't mean you by yourself. But together you're dangerous (like some chemical combination.' (PL: 317-318)

Sin embargo, Madame Merle no se amedrenta, y no sólo sigue adelante con su plan, sino que también intenta tomar el control de la vida de Pansy y así se lo hace ver a la tía paterna de la joven:

Madame Merle replied 'She'll soon be sixteen, and after that she'll begin to need a husband rather than a stepmother. 'And will you provide the husband as well?' 'I shall certainly take an interest in her marrying fortunately.' (PL: 321)

Ante la pregunta por parte de Mrs. Touchett, su vieja amiga, acerca de la supuesta relación de Isabel y Osmond, muestra un gran cinismo, que es si cabe, más aclaratorio sobre su culpabilidad en el desenlace de la historia:

'You who know everything,' [Mrs. Touchett] said, 'you must know this: whether that curious creature's really making love to my niece.' 'Gilbert Osmond?' Madame Merle widened her clear eyes and, with a full intelligence, 'Heaven help us,' she exclaimed, 'that's an idea!' 'Hadn't it occurred to you?' ¡You make me feel an idiot, but I confess it hadn't. I wonder', she added, 'if it has occurred to Isabel.' (PL. 325)
No obstante, esto no parece suficiente para ella, puesto que no sólo le miente sino que le promete que intentará saber algo más sobre el tema preguntándole a Osmond por sus intenciones. Muy al contrario, una vez que observa que su plan comienza a funcionar, no sólo se alegra tremendamente, sino que no deja de vigilar todos los detalles, así urge a Isabel para que vaya a Roma y descubre alegre que Osmond también lo hará, mientras ella se queda al cuidado de Pansy. Aunque atisbamos una pizca de remordimiento en una entrevista con Osmond antes de que el plan se ejecute por sí solo: “'I'm frightened at the abyss into I shall have cast her,' He took it almost gaily. 'You can't draw back - you've gone too far.' 'Very good; but you must do the rest yourself.' 'I shall do I,' said Gilbert Osmond" (PL: 335). Todo está hecho ya, ahora tan sólo cabe esperar el trágico desenlace, así acaba el primer volumen, mostrando una Serena Merle que parece arrepentida.

Sin embargo, los acontecimientos apuntan en otra dirección, el lector conoce las intenciones de Isabel de casarse con Osmond tras un viaje en el que Madame Merle ha jugado sus cartas de un modo maestro y cuando Mrs. Touchett es informada en el capítulo XXXIII de los planes de su sobrina, comprende quien es realmente Madame Merle, y es ella la que intenta hacer ver a Isabel la clase de persona que en realidad es su supuesta amiga, pero Isabel está cautivada no sólo por su futuro marido, sino también por Madame Merle y no puede ni quiere creer que es la víctima de un engaño tramado por sus dos seres más queridos. Isabel Archer, la víctima de tal crimen, será la última en conocer la verdad, por eso resulta interesante estudiar su reacción ante el cambio de los acontecimientos:

Aunt Lydia, I've something to tell you.' Mrs. Touchett gave a little jump and looked at her almost fiercely. 'You needn't tell me; I know what it is [ . . ] You're going to marry [ . I Madame Merle's friend (Mr. Osmond' 'I don't know why you call him Madame Merle's friend. 'If he's not her friend he ought to be (after wou call him Madame Merle's fiend. 'If he's not her 'she has doner it of her I'm disappointed.' If you mean that Madame Melle has had anything to do with my engagement you're greatly mistaken,' Isabel declared with a sort of ardent coldness.' (PL: 384)

But I shall talk to Madame Merle.' said Mrs. Touchett. 'I don't see why you keep bringing her in. She has been a very god to me'. 'Possibly; but she has been a poor one to me [...] She has deceived me. She had as good as promised me to prevent your engagement. [...] To me she has been honest and kind and devoted. "Devoted, of course, She wish watching you in order to interpose [...] She gained time, as you see. While I waited for her to interfere you were marching away, and she was really beating the drum.' (PL: 386) 
Tras su éxito, Madame Merle parece retirarse, de hecho existe un dato que sorprende al lector, Madame Merle no asiste a la ceremonia de la boda. ¿Ha acabado aquí el papel de Madame Merle? ¿Por qué su ausencia en la celebración de su victoria? ¿Qué razones le impiden disfrutar de su triunfo? También sabemos que ha roto su amistad con su vieja amiga, Mrs. Touchett, ¿se encuentra sola? "Madame Merle had chosen to pass many months in England [...] Madame Merle suffered in silence" (PL: 442). No obstante, años más tarde, la visita de un admirador de Pansy, Edward Rosier, reaviva su interés por la manipulación de la familia de Osmond, ya que éste le pide su ayuda para poder casarse con la chica. Madame Merle reacciona, y decide tomar parte en el futuro de Pansy a la vez que muestra celos de Isabel al afirmar que ella no tiene ningún derecho sobre la chica. Esto nos da muestras de lo lejos que está de Isabel, tal y como nos indica el narrador en el capítulo XL:

Isabel had not seen much of Madame Merle since her marriage, this lady having indulged in frequent absences from Rome. [...] Familiarity had modified in some degree her first impression of Madame Merle, but it had not essentially altered it it was admiration in it [...] Isabel now saw more of her than she has done since her marriage [...] She had lost the desire to know this lady's clever trick [...] Madame Merle was doubtless of great use to herself and an ornament to any circle [...] Madame Merle's cons of great use to herself and an ornament to any circle $[\ldots$ Madame Merle's conduct [...] was always "quiet". But in this matter of not wishing to intrude upon the inner life of the Osmond's family it at least occurred to ou young woman that she overdid a little $[\ldots]$ She remembered too much that Isabe was married [...] that though she, Madame Merle, had known Gilbert Osmond and his little Pansy very well, better almost than anyone, she was not after all of the inner circle. She was on her guard. (PL: 451-453)

A su vez, Isabel, tras tres años de matrimonio, ha tenido tiempo de reflexionar, y en alguna ocasión, ha recordado las palabras de su tía en relación con el rol que Madame Merle jugó en su compromiso con Gilbert, pero su soberbia no la deja ver que su libertad fue coartada sin ella ser consciente: "Madame Merle might have made Gilbert's marriage, but she certainly had not made Isabel's" (PL: 453). Sin embargo, algo preocupa a Isabel, y es la relación de su marido con esa dama misteriosa, no porque sienta celos, sino, una inmensa curiosidad por ese pasado oculto y vedado para ella. Mientras esto ocurre, Serena Merle reaparece en sus vidas, puesto que movida por su interés por recuperar su poder sobre Pansy, visita la casa de Osmond y mantiene con él largas e íntimas conversaciones. Isabel sufre su matrimonio, y descubre que entre ellos dos existe algo mágico, algo diferente.

Una escena entre Madame Merle e Isabel tiene lugar en un momento importante para el desenlace de la historia, y se sucede a propósito del interés que Lord Warburton un antiguo admirador de Isabel, muestra por casarse con Pansy:
I want to see her married to Lord Warburton.' 'You had better wait till he asks her.' 'If what you say's true, he'll ask her. Especially,' said Madame Merle in a moment, if you make him.' 'If I make him?' 'It's quite in your power. You've great influence with him [...] but if you wouldn't marry Lord Warburton yourself, make him the reparation of helping him to marry some one else. (PL: 462-463)

¿Es esto lo que ha ocurrido con Madame Merle? ¿Rechazó ella la petición de matrimonio por parte de Osmond y por eso quiso recompensarle arreglándole el matrimonio con Isabel? ¿Sigue enamorada Serena de Gilbert? ¿Qué comparten? ¿Qué sabe la condesa Gemini y esconce sobre ellos? Todas estas preguntas pueden ser las que Isabel se haga a partir de este capítulo. De hecho, nuestra joven protagonista empieza a obsesionarse con esto y sufre su verdadero drama:

More clearly than ever before Isabel heard a cold, mocking voice proceed from she knew not where, in the dim void that surrounded her, and declare that this bright, strong definite, worldly woman, this incarnation of the practical, the personal, immediate, was a powe had yet discovered, and her nearness was not the charming accident she had long supposed [...] No definite suspicion had as yet taken its place; but it was enough to make her view this friend with a different eye, $[\ldots]$ she seemed to wake from a long pernicious dream. (PL: 561)

Who are you (what are you?' Isabel murmured. 'What have you to do with my husband? [...] 'What have you to do with me?' Isabel went on. Madame Merle slowly got up, stroking her muff, but not removing her eyes fro Isabel's face. 'Everything!' she a back, covering her face with her hands, It had come over her like a high-surging wave that Mrs. Touchett was right. Madame Merle had married her. Before she uncovered her face again that lady had left the room. (PL: 564)

Es entonces cuando Isabel comienza a descubrir la realidad y comprende que su vida es quizá parte de un plan ajeno a ella y no el resultado de su propia libertad:

'There's something I want you to know,' the Countess declared 'because I think you ought to know it. Perhaps you do; perhaps you've guessed it. But if you have, all I can say is that I understand still less why you shouldn't do as you like.' [...] 'In your place I should have guessed it ages ago. Have your never really suspected?' I've guessed nothing. What should I have suspected? I don't know what your mean [...] Isabel slowly got up. 'You're going to tell me something horrible.' [...]'My first sister-in-law had no children [...] It wasn't till after her death that Pansy arrived.' [...] 'Pansy's not my husband 's child then?' 'Your husband's - in perfection! But no one else's husband's. Some one else's wife's' [...] 'Whose wife's?' Isabel asked. "The wife of a horrid little Swiss who died. He never recognized Miss Pansy [...] Osmond did. [. The story passed, sufficiently; it was covered by the appearances Osmo so long as nobody heeded, as nobody carse, I knew [...] She had spoken no name, yet Isabel could but check, on her own lips, an echo of the unspoken $[\ldots]$ 'Had it never occurred to you that he was for six or seven years her lover?' (PL: 587-589) 
Sin embargo, en el clímax de la novela, cuando Isabel se enfrenta a la verdad en el capítulo LI, su reacción es distinta a la que quizá espera el lector. La heroína reacciona mostrando pena por Madame Merle porque entiende que la verdadera víctima no es ella si no quien buscando unir su vida a la de Osmond ha descubierto que el destino no le da lo que en principio prometía “'Poor woman_and Pansy who doesn't like!' cried Isabel" (PL: 591). Madame Merle por el contrario, al verse descubierta, se ve obligada a continuar con su papel, y decide intentar un nuevo plan al descubrirle a Isabel que no sólo debe agradecerle a ella su desgracia, sino que su primo Ralph ha sido el verdadero artífice del desenlace puesto que sin su intervención en la decisión del difunto Mr. Touchett en cuanto a la herencia, Isabel nunca hubiera sido un objetivo ni para ella ni para Osmond. Madame Merle intenta hacer cómplice de sus manipulaciones a Ralph Touchett, pero tal y como argumenta Craig Howard White "[t]he supposed difference is that Madame Merle, self-interested, has deliberately lied to or withheld the truth from Isabel, while Ralph, dis-interested, has had her best interests at Herat" (1991: 205) ${ }^{6}$. Tras esta última trasgresión, decide desaparecer, y lo hace bajo un halo de misterio, porque ni el lector ni Isabel saben si lo hace para exiliarse y de algún modo expurgar su culpa o para seguir cometiendo nuevas fechorías en tierras más permisivas donde la sociedad europea no la margine, ni la juzgue.

\section{LA REALIDAD DE MADAME MERLE: ESTUDIO SINCRONIICO DE LA INFOR- MACIÓN}

El segundo análisis que realizamos de la figura de Madame Merle es un estudio sincrónico, es decir, un estudio en el que observamos toda su realidad y consideramos a la vez toda la información de la prehistoria y la historia. Así llegamos a descubrir que no es tan sólo uno de los dos personajes malvados y desencadenantes del mal de la novela, sino que realmente es sólo una víctima, y que ha sido abocada a desempeñar ese papel en la trama. Intentaremos demostrar quién o qué es entonces el agente opresor que convierten a la figura malvada de la obra en una de las vícitmas. Es decir descubriremos el proceso de victimización que sufre este personaje de la novela y cambiaremos así la división maniqueísta del texto.

En la prehistoria de la novela descubrimos a una Madame Merle casada con un hombre europeo aunque no por ello feliz, puesto que está enamorada de otro hombre, Gilbert Osmond. Tenemos pues, ya una víctima, una mujer que sufre por amor y por la presión social, ya que éste es el primer dato de su posible marginalización social

6 Sin embargo, tal y como se apunta en este trabajo, Madame Merle no es tan 'self-interested' como se hace ver, puesto que como a continuación se intenta demostrar no es más que una víctima. En cuanto a la actuación desinteresada de Ralph Touchett, quizá habría que tener en cuenta factores que en este análisis quedan fuera de contexto, pero sería interesante promulgar una inocente duda ante esta división tan clara entre los protagonistas de la novela. por parte de un grupo de personajes de la alta sociedad europea que no aceptan el adulterio de una mujer americana que intenta encontrar su sitio en el viejo continente. Adentrándonos en su affaire con Osmond, conocemos también que él es un hombre casado con una joven, que sin embargo muere tras tres años de matrimonio y $\sin$ haber tenido descendencia. El adulterio entre Gilbert y Serena ha durado entre seis o siete años y de ese amor prohibido ha nacido una niña ilegítima que por supuesto, no fue nunca reconocida por el marido de Madame Merle pero sí por su propio padre. Tras la muerte de la primera mujer de Osmond, la niña ha pasado a manos de éste que ejerce su papel de padre al estilo de la época, es decir, enviando a una niña a un convento ${ }^{7}$ para que otras figuras femeninas sustituyan a la figura materna. Asi entendemos que Madame Merle renuncia a todos los derechos sobre la niña para que pueda disfrutar de un apellido, una situación social, una buena educación y una familia de reconocido prestigio. Tras la ruptura con Osmond, Madame Merle vive como una viuda desarraigada de todo, puesto que no tiene familia, marido o amante, hijos, ni una patria a la que volver. Acercándonos ya a la propia historia de la novela observamos que han pasado doce o quince años desde que todo esto ocurrió y que Madame Merle es una madre oculta que mantiene secretos y oscuros lazos con el padre de su hija y que movida por este amor en la sombra empieza a jugar un papel moviendo los hilos necesarios para dotar a su hija, y en consecuencia, al que un día fue su amante, de una vida llena de lujo y felicidad. Por ello, en cuanto conoce a Isabel decide unir su vida a la de sus dos y únicos seres queridos. Madame Merle es a la vez, amiga, madre y amante cuando lucha por precipitar el matrimonio entre Isabel y Gilbert, porque cree que está ofreciendo por un lado, una madre, una esposa y dinero a su hija y a su antiguo amor, y por otro, una hija y un marido a Isabel. Bajo su personal punto de vista, está proporcionando LA FELICIDAD a todos ellos, porque es eso lo que seguramente ella no pudo tener ni disfrutar, y lo que la ha hecho infeliz, pero no sabe ver que Isabel es un alma libre y que nada la hará sufrir tanto como vivir lo que otros han decidido por ella. En este sentido, Madame Merle es claramente una víctima de una sociedad que la ha privado de un amante y de una hija, y a su vez, la ha obligado a precipitar la tragedia de otro personaje ajeno a todo esto. A lo largo de toda la novela, observamos las críticas de varios personajes de la sociedad europea hacia su figura, tanto su nacionalidad como su comportamiento y su pasado son objetivo de los más duros y ácidos comentarios por parte de la gran parte de esta sociedad, que aunque, en cierto modo, la respeta, no la acepta. Así pues, el primer agente opresor en este proceso de victimización del personaje de Madame Merle es el que juega la sociedad del continente europeo que rivaliza con ella, y que precisamente al final de la novela

7 Recordemos de nuevo las connotaciones que este tipo de instituciones tienen en Europa, y tengamos en cuenta que el deseo por parte de Osmond de que su hija permanezca en el convento se opone a los deseos de Madame Merle, quien cree que la niña es demasiado pura y natural para permanecer en un lugar como ése. 
se libera de ella, puesto que como sabemos, Madame Merle decide abandonar Europa y regresar a América. Esta huída puede ser interpretada como un exilio obligado tras sus artimañas, pero también, como una liberación del personaje ante la presión de una sociedad que de un modo cínico no permite la verdadera libertad de la mujer 'moderna'.

Por otro lado, podemos afirmar que la figura masculina ejerce también un papel dominante en este proceso de victimización femenina, puesto que el amor que Madame Merle siente por Gilbert Osmond es el verdadero desencadenante de toda la tragedia. ¿Sigue amando Madame Merle a Osmond? Aunque no fuese así, todo lo que busca ella es la felicidad de éste y de su hija, y no bajo su colaboración ni su respeto. Ella lucha en soledad, al final de la historia, Madame Merle ha perdido el amor de Pansy, el amor de Gilbert, la amistad con Isabel, con Mrs. Touchett y la poca aprobación de la que gozaba al principio por algún sector de esa sociedad en la que se empezaba a aclimatar, as lo muestran los diálogos de muchos de estos personajes en la última parte de la obra:

'Don't you like Madame Merle?' Isabel had once said to him. 'She thinks a great deal of you.' 'I'll tell you once for all,' Osmond had answered. 'I liked her once better than I do today. I'm tired of her, and I'm rather ashaned of it. She's so almost unnaturally good! I'm glad she's not in Italy; it makes for relaxation - for a sort of more détente. Don't talk of her too much; it seems to bring her back. She'll come back in plenty of time'. (PL: 454)

'I don't believe you're unhappy; I believe you like it,' said Madame Merle. 'Did I say I was unhappy?' Osmond asked with a face grave enough to suggest that he might have been. 'No, but you don't say the contrary, as you ought in common might he' 'Don't talk about gratitude,' he returned dryly. 'And don't aggravate gratitude.' 'Don't talk but you don't say the contrary, as you ou don't aggravate me,' he added in a moment [..] "I'm very tired." "Eh mly. 'And don!" cried Madame Merle [...] 'When I fatigue myself it's for you. I've given you an interest. That's a great gift'. (PL: 567)

Sister Corona Sharp ofrece un magnifico párrafo que muestra claramente el sufrimiento de la víctima que Madame Merle encierra, y aunque Sharp no admite este proceso de victimización, sus comentarios sí lo hacen

Her frustrations are more numerous than those of other confidantes: a loveless marriage; an episode in another man's life, from which she could emerge only as the loser; the sacrifice of her child for the sake of respectability; the failure to contract a brilliant, second marriage -all these are truly great disappointments. Ironically, pity is accorded to her only by her victim [...] The man for whom she has ronically, pity is accin she has worked, plotted, and suffered, makes no secret of his being tired of her. Nothing, indeed, is spared her; and one is forced to admire the pride that can hold her head
erect to the very end. (1963: 95)
Ya que tal y como apunta Stafford "[...] this compelling summation is hardly less appropriate as a description of Isabel's fate that it is of Madame Merle's [...]" (1986: $120)^{8}$.

Pero lo que más atormenta a Serena en los momentos finales es descubrir que ha luchado para no lograr nada: Osmond tiene dinero pero es infeliz, Pansy no parece encontrar su camino, Isabel la rechaza y nadie muestra gratitud, sino más bien lo contrario. "She has found a wife for Osmond, but Osmond has never lifted a little finger for her. She has worked for him, plotted for him, suffered for him; she has even more than once found money for him; and the end of it is that he's tired of her" (PL: 594). Su grito atormentado, en el capítulo XLIX, refleja todo el dolor y sufrimiento de una mujer que ha luchado en vano, que al final entiende que debe cerrar un ciclo, que debe abandonar su intento, y que realmente se encuentra sola e infeliz: 'Have I been so vile all for nothing!' (PL: 571).

\section{CONCLUSIÓN}

'Have I been so vile all for nothing!' Es difícil no reconocer la víctima que encierra este grito, este desenlace. Sin embargo, pocos son los críticos y los estudios que abordan el tema de la victimización femenina en la obra, ocupándose de Madame Merle como el objeto de dicho proceso. Brillante excepción es el trabajo de William Stafford, ya citado con anterioridad en este artículo, que apunta dos puntos de vista a la hora de analizar la misteriosa y compleja figura de Madame Merle "[f]rom one point of view, she is simply the designing villainess who is finally exposed and gets her predictable comeuppance [...] But from another point of view, is it not Serena Merle who has come through the whole less scarred than Isabel herself? It is perhaps even Serena Merle, through the loss of the love of her child, who most poignantly suffers"' (1986: 121).

Isabel Archer es evidentemente la víctima del plan tejido por aquella, pero no debemos olvidar que el resultado perjudica más a Madame Merle que incluso a la primera protagonista femenina. A lo largo de estas páginas se ha intentado demostrar que el personaje de Serena Merle sufre en la obra un proceso de victimización por parte de varios agentes. En primer lugar destacamos el lugar que la sociedad europea ha ocupado en dicho proceso, por medio de la marginalización que se lleva a cabo de este personaje femenino nacido en el continente americano y que por falta de pasado -al igual que el continente del que procede- ascendencia aristócrata, dinero y relaciones, se ve abocada a la soledad y al juicio público. En segundo lugar, hemos señalado y analizado el rol masculino que desarrolla Gilbert Osmond, amparado por una sociedad

8 Quizá en la similitud entre los dos personajes femeninos encontramos la clave de la identificación de Madame Merle con la otra víctima del drama. Por otro lado, esta comparación entre ellas, junto al dato apuntado aquí anteriormente acerca de la indirecta culpabilidad de Ralph Touchett en el desenlace final, supone también la ruptura de la división maniqueísta de los personajes de la novela. 
patriarcal que permite el meretricio del género femenino. Por último, podemos afirmar, que la técnica narrativa escogida por James para la novela proporciona también una perspectiva que resulta perniciosa para la figura de Madame Merle, puesto que la dosificación de información, el juego realizado con los satélites de la narración y e desenlace brusco de la parte final de la novela, perjudican el juicio que el lector hace de Madame Merle, ya que como se ha demostrado con la comparación entre el análisis cronológico y el sincrónico, nuestra opinión acerca de ella cambia según se observe de un modo u otro. Como anticipábamos al inicio de este artículo Madame Merle es un personaje mucho más rico y complejo de lo que en muchas ocasiones se ha entendido, el propio Henry James en sus Notebooks afirma que "[...] the strangeness of her conduct is greater; but we must remember that we see only its surface -we don't see her reasoning" (1947: 17). Así pues, insistiendo en esta idea del propio James acerca de la superficialidad con la que este personaje es tratado en la obra, quizá necesitásemos para entender mejor la riqueza y complejidad de esta dama, que el punto de vista de la narración no oscilase tan sólo entre el de Isabel y los demás satélites, sino que se centrase más en el de Madame Merle, y así podríamos encontrar muchas, o quizá todas las respuestas a las preguntas aquí planteadas.

\section{REFERENCIAS BIBLIOGRÁFICAS}

Armorial Gold Heraldry Services. Heraldryclipart.com. 6-10-04. http://www.heraldryclipart.com/ heraldry-symbolism.html

Beach, J. W., The Method of Henry James, New Haven: Yale U.P., 1918.

Collins, M., "The Narrator, the Satellites, and Isabel Archer: Point of View in The Portrait of a Lady" Studies in the Nove, 18 (1976), pp. 142-57.

Foss, C., "Female Innocence as Other in The Portrait of a Lady and What Maisie Knew: Reassessing the Feminist Recuperation of Henry James", Essays in Literature, 22.2 (1995), pp. 253-68.

James, H., The Notebooks of Henry James, ed. F. O. Matthiessen, y K. B. Murdock, New York: Oxford U.P., 1947.

---, The Portrait of a Lady (1881), ed. Geoffrey Moore, London: Penguin, 1984.

Poole, A., "Dying before the End: The Reader in The Portrait of a Lady", Yearbook of English Studies, 26 (1996), pp. 143-53.

Sayres, W. G., “The Proud Penitent: Madame Merle's Quiet Triumph in Henry James's The Portrait of a Lady" Essays in Literature, 19.2 (1992), pp. 231-45.

Sharp, S. C., The Confidante in Henry James: Evolution and Moral Value of a Fictive Character, Notre Dame: University of Notre Dame Press, 1963.
Solomon, M., "The Female World of Exorcism and Displacement (or, Relations between Women in Henry James's Nineteenth- Century The Portrait of a Lady)", Studies in the Novel 28.3 (1996), pp. $395-413$

Stafford, W. T., “The Enigma of Serena Merle”, The Henry James Review, 7.2-3 (1986), pp. 117-123. White, C. H., “The House of Interest: A Keyword in The Portrait of a Lady”, Modern Language Quarterly: A Journal of Literary History, 52.2 (1991), pp. 191-207. 